\title{
Using Gold Nanoparticles with Spectroscopic and Analytical Models for Rapid Colorimetric Detection of Mercury Ions in Aqueous Media
}

\author{
Jun HE ${ }^{1,2,3}$, Zihan WU ${ }^{1,2,3}$, Ming SUN ${ }^{1,2,3 *}$ \\ ${ }^{1}$ College of Information and Electrical Engineering, China Agricultural University, Beijing 100083, China \\ ${ }^{2}$ Key Laboratory of Agricultural Information Acquisition Technology, Ministry of Agriculture, Beijing 100083, China \\ ${ }^{3}$ Beijing Engineering and Technology Research Center for Internet of Things in Agriculture, Beijing 100083, China \\ crossref http://dx.doi.org/10.5755/j01.ms.25.2.19140
}

Received 27 September 2017; accepted 09 April 2018

\begin{abstract}
In this study, high-sensitively colorimetric detection of mercury ions was developed according to the surface plasmon resonance of modified gold nanoparticles. While in a citrate buffer solution, the selected modifier Bismuthiol II can cause gold nanoparticles to aggregate. Gold nanoparticles with a spherical shape and average diameter of about $13 \mathrm{~nm}$ were prepared. While mercury ions can inhibit gold nanoparticle aggregation, after the addition of mercury ions, the color of the gold nanoparticles solution changes from light violet to red with a hypochromatic shift at the absorption peak. By collecting the UV-Vis spectrum of the solution after the reaction, the characteristic bands are selected to build a partial least squares model. Both the 0.9477 correlation coefficient with a $0.0986 \mathrm{mg} / \mathrm{L}$ standard deviation for the calibration set, and 0.9274 correlation coefficient with a $0.1020 \mathrm{mg} / \mathrm{L}$ standard deviation for the prediction set were obtained via a partial least squares model.

Keywords: gold nanoparticles, colorimetry, mercury ions, spectroscopy.
\end{abstract}

\section{INTRODUCTION}

There are three forms of mercury in nature: elemental, ionic, and organic mercury; each is a transformation of the other. Mercury ions $\left(\mathrm{Hg}^{2+}\right)$ are able to pass through the skin, intestinal tract, and respiratory tissue of the human body, which can damage the nervous system, immune system, kidneys, liver, and even lead to hearing and visual impairment [1,2]. Therefore, mercury has been classified as a carcinogenic agent, so the World Health Organization restricted mercury ions levels in drinking water to lower than $2 \mu \mathrm{g} / \mathrm{L}$ [3]. Detecting heavy metal pollution has been a serious subject for research scientists, and it has consumed a significant amount of human, material, and financial resources each year. Due to environmental pollution, there is an urgent need for convenient, real-time, on-site analysis methods. In recent years, gold nanoparticles (AuNPs) have drawn remarkable interest in the application of mercury ion detection due to their strong absorption of electromagnetic waves in the UV-Vis region due to the Surface Plasmon Resonance (SPR), highly stable dispersions, chemical inertness, and biocompatibility, which make them easily adaptable to biosensor components $[4,5]$. The gold nanoparticle solution presents wine red; the solution turns purple-blue when the distance between particles decreases, solution turns to purple blue, which results in the coupling of surface plasmon resonance, between particles in close proximity. As the distance between particles decreases or the particles size increases, the effect of plasmonic coupling increases, resulting in a red shift of the plasmon band in the UV-Vis spectrum and a change in solution color $[6,7]$. When a heavy metal or another specific

\footnotetext{
* Corresponding author: Tel.: +86-135-2055-7807.

E-mail address: sunming@cau.edu.cn (M. Sun)
}

analyte is introduced, the solution color visibly changes with the aggregation state of biologically functionalized gold nanoparticles in the solution, which is the theoretical basis for colorimetric biosensors that use gold nanoparticles [8].

Common detection methods include atomic spectrometry, electrochemical analysis, biological analysis, and so on. Atomic spectrometry contains Atomic Absorption spectrometry (AAS), Inductively Coupled Plasma Atomic Emission Spectrometry (ICP-AES), Atomic Fluorescence Spectrometry (AFS), and has high sensitivity, but the sample pretreatment and testing process is relatively complex, and it relies on the large-scale equipment, requires specialized technical personnel to operate, is time-consuming, and has difficulty in fast-field detection [9-11]. Electrochemical analysis quickly detects heavy metals with portable instruments, but has low sensitivity and accuracy, is expensive, and is limited in the kinds of heavy metal that it can detect [12-17]. Biological analysis methods include the enzymatic inhibition method, immunoassay, and so on; they are suitable for the qualitative detection of heavy metals; however, the sensitivity and accuracy also requires further research. Compared to these common methods, UV-Vis spectrophotometry has better sensitivity and a lower detection limit, all while having the following benefits: it is simple to operate, has a short reaction time, and is noncorrosive and non-pollutive. In addition, compared to traditional reagent colorimetric methods, the gold nanoparticles colorimetric method has a very high molar absorption coefficient of gold nanoparticles, which is higher than that of organic reagent by about 3 to 5 orders of magnitude. In this study, gold nanoparticles were modified by Bismuthiol II to quickly detect $\mathrm{Hg}^{2+}$ in water in a short time with high selectivity and sensitivity. Stable 
gold nanoparticles of uniform particle size were easily acquired. At the same time, Bismuthiol II had the advantages of a lower price and no pollution emission.

\section{MATERIALS AND METHODS}

\subsection{Materials}

Chloroauric acid $\left(\mathrm{HAuCl}_{4} \cdot 4 \mathrm{H}_{2} \mathrm{O}\right)$ and Bismuthiol II $\left(\mathrm{C}_{8} \mathrm{H}_{5} \mathrm{KN}_{2} \mathrm{~S}_{3}\right)$, which served as gold nanoparticle modifiers in this experiment, were obtained from Xiya Reagent (Chengdu, China). Trisodium citrate $\left(\mathrm{Na}_{3} \mathrm{C}_{6} \mathrm{H}_{5} \mathrm{O}_{7} \cdot 2 \mathrm{H}_{2} \mathrm{O}\right)$ and sodium hydroxide $(\mathrm{NaOH})$ were procured from Xilong Co.Ltd (Beijing, China). Citric acid $\left(\mathrm{C}_{6} \mathrm{H}_{8} \mathrm{O}_{7}\right)$ was purchased from Beijing Chemical Works (Beijing, China). The mental salt solutions were obtained from the Institute for Environmental Reference Materials Ministry of Environment Protection, China. Deionized water was purchased from Yixiubogu Co. Ltd (Beijing, China). The deionized water and the chemicals used for the study were used following standard procedures.

\subsection{Instruments}

The UV-Vis spectroscopy was recorded on a UV-2450 spectrophotometer with $0.1 \mathrm{~nm}$ resolution from Shimadzu Co. Ltd (Japan). Transmission electron micrographs (TEM) of gold nanoparticles were obtained on a JEM-1230 electron microscope from JEOL Co. Ltd (Japan). RP1003H electronic balance and 78-1 magnetic heated stirrers were needed in the solution preparation process.

\subsection{Synthesis of gold nanoparticles using the citrate method}

There are many methods to acquire gold nanoparticles. In this study, gold nanoparticles were obtained using citric acid to revivify chloroauric acid. A $100 \mathrm{~mL}$ solution of $0.01 \%$ chloroauric acid was heated to its boiling point, and then $4 \mathrm{ml}$ of $1 \%$ trisodium citrate was rapidly added and stirred in the boiled solution. The solution color changed from pale yellow to colorless; two minutes later, it changed to blue-black, then purple-black, and steadily shifted to red. Then, the mixture was cooled to room temperature, and deionized water was added to $100 \mathrm{ml}$ as the original volume. In this reaction, trisodium citrate acted as a reducer, which has a relatively stable reduction capacity and protects the gold nanoparticles solution from precipitating during the chloroauric acid reducing process. The gold nanoparticle aggregation was clear and transparent, did not have any impurities, and precipitated into a wine-red.

\subsection{Experimental procedure}

The citric acid-sodium citrate was prepared with a $\mathrm{pH}$ of 4.4 as a buffer solution. First, $70 \mu 1$ of a buffer solution was added in a centrifuge tube $(10 \mathrm{ml})$. Then, $1.5 \mu \mathrm{mol} / \mathrm{L}$ of Bismuthiol II $(100 \mu \mathrm{l})$ was added to the tube and the solution was thoroughly mixed via shaking. Then, after adding a $\mathrm{Hg}^{2+}$ solution with various concentrations ranging from $0.05 \mu \mathrm{g} / \mathrm{L}$ to $1.00 \mathrm{mg} / \mathrm{L}$, the tube was shaken for 10 minutes. Finally, the gold nanoparticle solution was mixed in the solution, and 30 minutes later the spectra from $400 \mathrm{~nm}$ to $800 \mathrm{~nm}$ was collected by the Shimadzu UV-Vis spectrophotometer.

Software Matlab2012 was employed in data processing. Matlab is a data analyzing and processing tool with an efficient visualization algorithm. There was a significant amount of noise in the raw spectra due to the interference of electronic noise, light, instrument performance, and other factors. To establish a stable mathematical prediction model, the original spectra must be pretreated and feature bands must be extracted to eliminate or reduce the influence from irrelevant factors [18]. In this study, a variety of pretreatment methods on original spectral data was employed, and then Partial Least Squares regression (PLS regression) models were established to choose the best pretreatment method according to the prediction accuracy of these models. Partial least squares regression is a multivariate statistical data analysis method, and mainly studies regression involving multiple dependent variables and arguments, especially when there are highly correlate relations between variables. In partial least squares regression, the spectral information matrix $X$ and the concentration matrix $Y$ were decomposed, and the formula is:

$$
\begin{aligned}
& Y=U Q^{T}+E_{Y}=\sum_{k=1}^{f} u_{k} q_{k}^{T}+E_{Y}, \\
& X=T P^{T}+E_{X}=\sum_{k=1}^{f} t_{k} p_{k}^{T}+E_{X},
\end{aligned}
$$

where $t_{k}(n \times 1)$ and $p_{k}(1 \times m)$ are the scores and loads of the kth principal component of the spectral information matrix $X$, respectively, $u_{k}(n \times 1)$ and $q_{k}(1 \times m)$ are the scores and loads of the kth principal component of the spectral information matrix $Y$, respectively, $f$ is number of the principal component. That is, $T$ and $U$ are scoring matrices for the $X$ and $Y$ matrices, respectively, $P$ and $Q$ are their loading matrices, respectively, and $E_{X}$ and $E_{Y}$ are their partial least squares regression fitting residual matrices. Then, the scoring matrices $T$ and $U$ were linearly regressed:

$$
\begin{aligned}
& U=T B, \\
& B=\left(T^{T} T\right)^{-1} T^{T} Y .
\end{aligned}
$$

The predicted concentration $Y_{P}$ of test sample was calculated as:

$$
Y_{P}=T_{P} B Q,
$$

where $T_{P}$ is scoring matrices for the $X$ matrices of test sample.

The Savitzky-Golay (SG) convolution smoothing method reduces noise by filter function by the least fitting squares. The derivative can enhance weak spectra information, and also extract effective and useful information. Using a polynomial to calculate the smoothed average value at wavelength $k$ :

$$
x_{k, \text { smooth }}=\bar{x}_{k}=\frac{1}{H} \sum_{i=-\omega}^{+\omega} x_{k+i} h_{i},
$$


where $h_{i}$ is SG smoothing factor, $H$ is normalization factor, $H=\sum_{i=-\omega}^{+\omega} h_{i}$.

Multiplicative scatter correction (MSC) and Standard Normal Variate (SNV) are both used to correct the spectral light shift caused by scatter on the non-uniformity sample surface. SNV can also weaken the effect of the optical path change and surface scattering [19]. SNV is processing a row vector spectral data:

$$
x_{S N V}=\frac{x-\bar{x}}{\sqrt{\frac{\sum_{k=1}^{m}\left(x_{k}-\bar{x}\right)^{2}}{m-1}}},
$$

where $\bar{x}=\left(\sum_{k=1}^{m} x_{k}\right) / m, m$ is the number of wavelength point, $k=1,2, \ldots, m$.

Unlike SNV, MSC is processing a set of spectral data.

\section{RESULTS AND DISCUSSION}

\subsection{Characterization of gold nanoparticles and detection mechanism of mercury ions}

Gold nanoparticles have strong surface plasmon resonance, absorption within UV-Vis spectra, and their bright color could be observed with a naked eye. Fig. 1 shows the result of the spectral scan of the gold nanoparticle solution on the spectrophotometer with a wavelength ranging from $400 \mathrm{~nm}$ to $800 \mathrm{~nm}$. As can be seen from the figure, the absorption peak is around $530 \mathrm{~nm}$ with a sharp shift, which indicates that the gold nanoparticles are uniform in size. Transmission electron microscopy is a powerful tool for characterizing gold nanoparticles, which can visually obtain the size and shape of gold nanoparticles. Fig. 2 represents the transmission electron micrographs (TEM) image of freshly synthesized gold nanoparticles and the aggregation state of gold nanoparticles-Bismuthiol II. Transmission electron micrographs analysis revealed that the gold nanoparticles were spherical, and their average diameter was around $13 \mathrm{~nm}$. The upper right corner of Fig. 2 shows the color of the solution changing from red to light violet with the addition of Bismuthiol II to the gold nanoparticle solution. Fig. 3 shows the size distribution of gold nanoparticles in the TEM image. Transmission electron micrograph (TEM) images of newly synthesized gold nanoparticles are smoothed, segmented, area labeled, and feature extracted to obtain a size distribution histogram of gold nanoparticles. Each number in the $\mathrm{X}$ axis stands for a gold nanoparticle, while the $\mathrm{Y}$ axis corresponds to the size of gold nanoparticles. The size of most of the gold nanoparticles is similar.

The sulphur atom of Bismuthiol II and the gold atom of gold nanoparticles can form an Au-S bond. The Au-S bond is stronger than that of the gold atom and the carboxyl of the sodium citrate on the surface of the original gold nanoparticles; thus the Bismuthiol II substitute induces gold nanoparticle aggregation, as the solution color changes from red to light violet, with a red shift of the absorption spectrum.

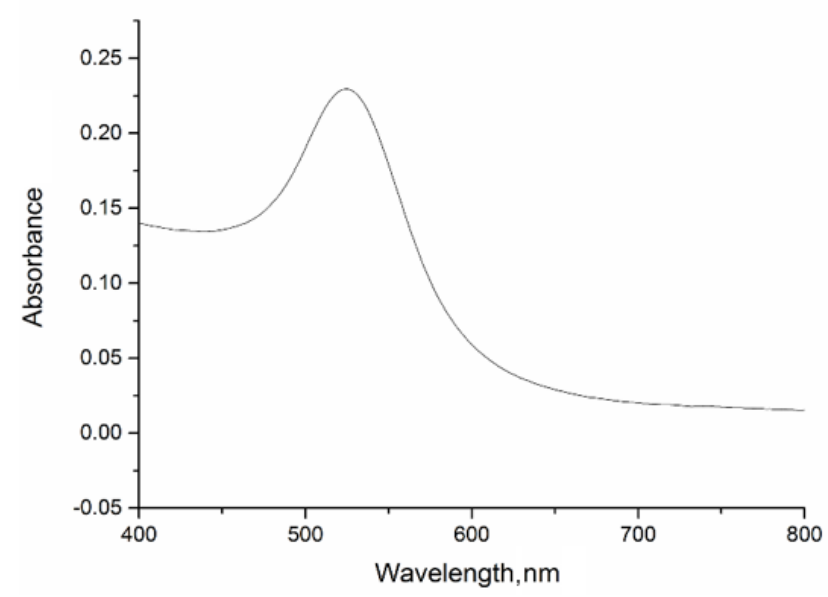

Fig. 1. UV-Vis spectra of the solution with gold nanoparticles

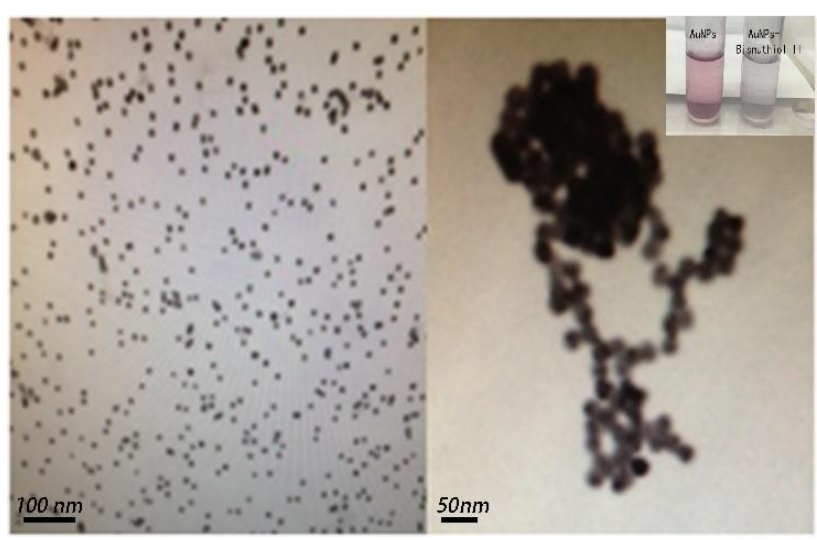

Fig. 2. Transmission electron micrographs image of gold nanoparticles and gold nanoparticles with Bismuthiol II

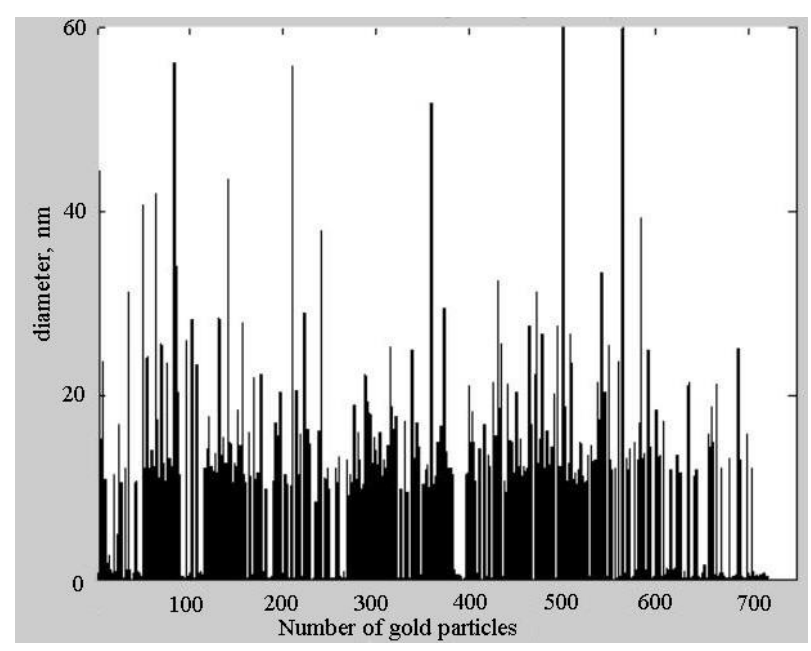

Fig. 3. The size distribution histogram of gold nanoparticles

With the addition of $\mathrm{Hg}^{2+}$ in the solution, Bismuthiol II combines with $\mathrm{Hg}^{2+}$ in an $\mathrm{Hg}-\mathrm{S}$ bond, which is stronger than the Au-S bond with a red color. As the $\mathrm{Hg}^{2+}$ concentration increases, more Bismuthiol II will be combined with $\mathrm{Hg}^{2+}$, and less Bismuthiol II with gold nanoparticles, which inhibits gold nanoparticle aggregation [20]. As shown in Fig. 4, a characteristic absorbance of gold nanoparticles was observed at about 530nm; as the 
$\mathrm{Hg}^{2+}$ addition increased, the absorbance intensity at $530 \mathrm{~nm}$ increased while that at approximately $650 \mathrm{~nm}$ declined, demonstrating that $\mathrm{Hg}^{2+}$ can induce the disaggregation of complex gold nanoparticles and Bismuthiol II.

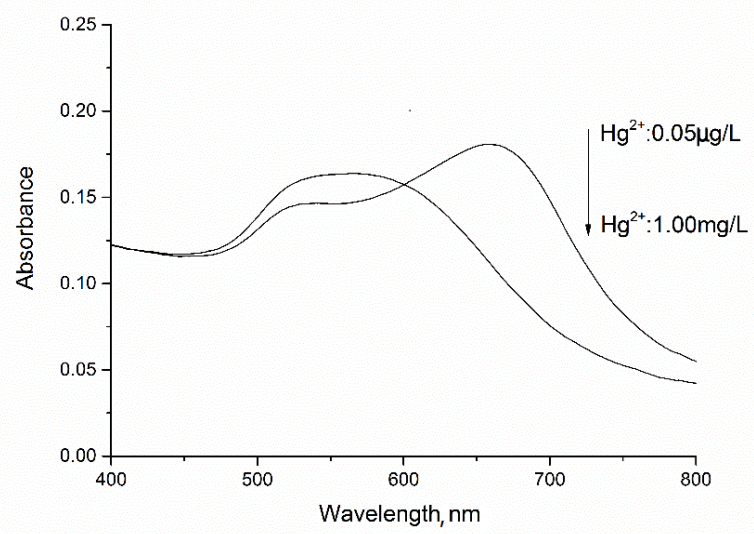

Fig. 4. UV-visible spectra of gold nanoparticles with Bismuthiol II solution after the addition of mercury ions from the concentration of $0.05 \mu \mathrm{g} / \mathrm{L}$ to $1.00 \mathrm{mg} / \mathrm{L}$

The color change from light violet to wine-red indicated a dispersed state of gold nanoparticles. Fig. 5 shows the different concentrations of Bismuthiol II affects the aggregation degree and absorbance of gold nanoparticles.

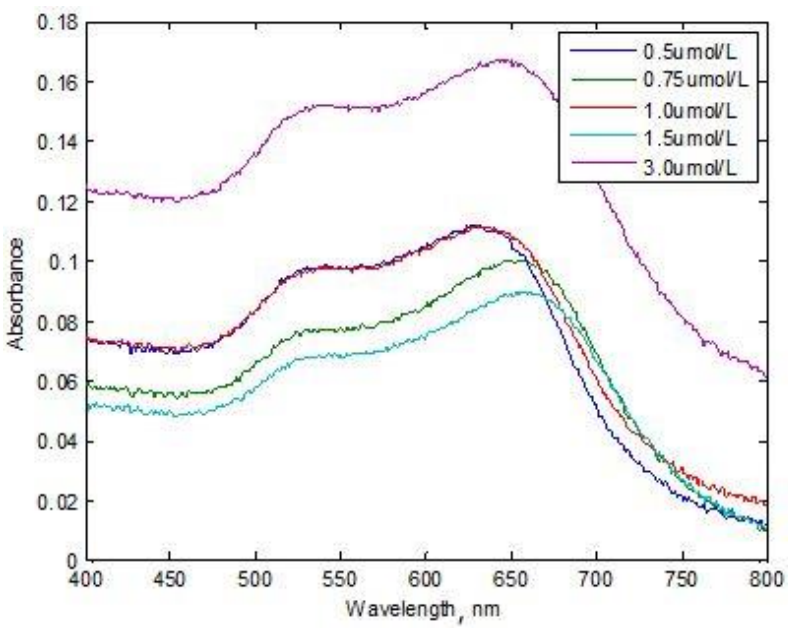

Fig. 5. UV-visible spectra of gold nanoparticles with different concentrations of Bismuthiol II

In the case of adding $3.0 \mu \mathrm{l}$ of Bismuthiol II to the gold nanoparticles solution, the measured spectral curves have peaks at $530 \mathrm{~nm}$ and $650 \mathrm{~nm}$, and the absorbance at $650 \mathrm{~nm}$ is higher than $530 \mathrm{~nm}$. According to the characteristic plasma resonance effect of gold nanoparticles, a large number of gold nanoparticles gathered. Bismuthiol II combines with $\mathrm{Hg}^{2+}$ in an $\mathrm{Hg}-\mathrm{S}$ bond, which is stronger than the $\mathrm{Au}-\mathrm{S}$ bond with a red color. Due to the excessive modifier Bismuthiol II, more mercury ions need to be combined to achieve the purpose of inhibiting the aggregation of gold nanoparticles, thereby reducing the sensitivity of mercury ion detection. And too few modifiers will reduce the entire detection range, while the color change is not obvious. When the concentration of modifier is $1.5 \mu \mathrm{mol} / \mathrm{L}$, the color range of gold nanoparticles solution is wide and suitable for observation, and it is suitable for detecting low concentration of mercury ions. As shown in Fig. 6, when the buffer volume increased from $40 \mu 1$ to $80 \mu \mathrm{l}$, the absorbance of the gold nanoparticles increased, and the absorbance at the peak at $520 \mathrm{~nm}$ and $650 \mathrm{~nm}$ is getting closer. Indicating that the buffer to a certain extent can cause the aggregation of gold nanoparticles. Too small citric acid-sodium citrate as a buffer solution cannot play the role of $\mathrm{pH}$ value. But too much buffer will cause a large number of gold nanoparticles aggregation, thus affecting the detection of mercury ions. So using $70 \mu \mathrm{l}$ volume of the buffer, which has little effect on the degree of aggregation of gold nanoparticles and absorbance is relatively large.

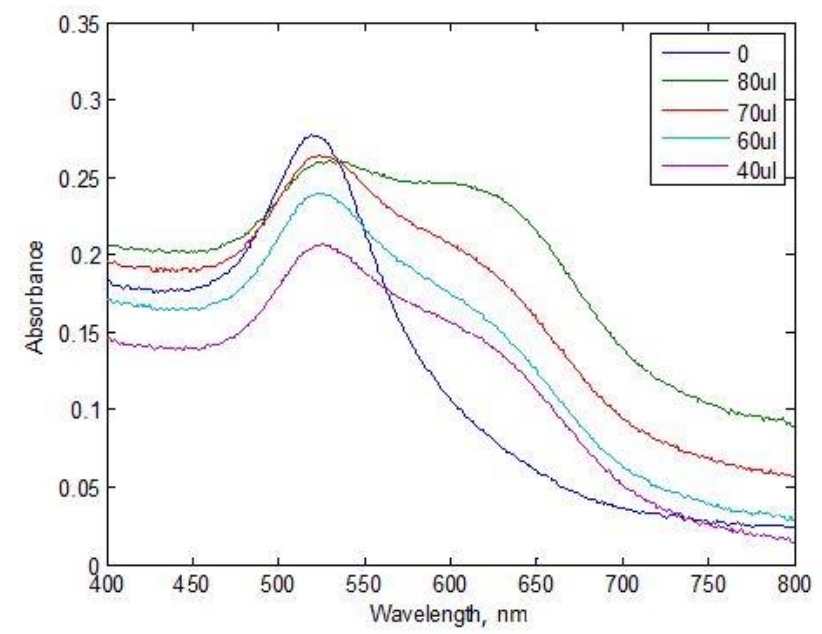

Fig. 6. UV-visible spectra of gold nanoparticles with different volume buffer

\subsection{Spectra data pre-processing}

Fig. 7 to Fig. 12 represents the results of the different pretreatment methods for spectral data from $400 \mathrm{~nm}$ to $800 \mathrm{~nm}$.

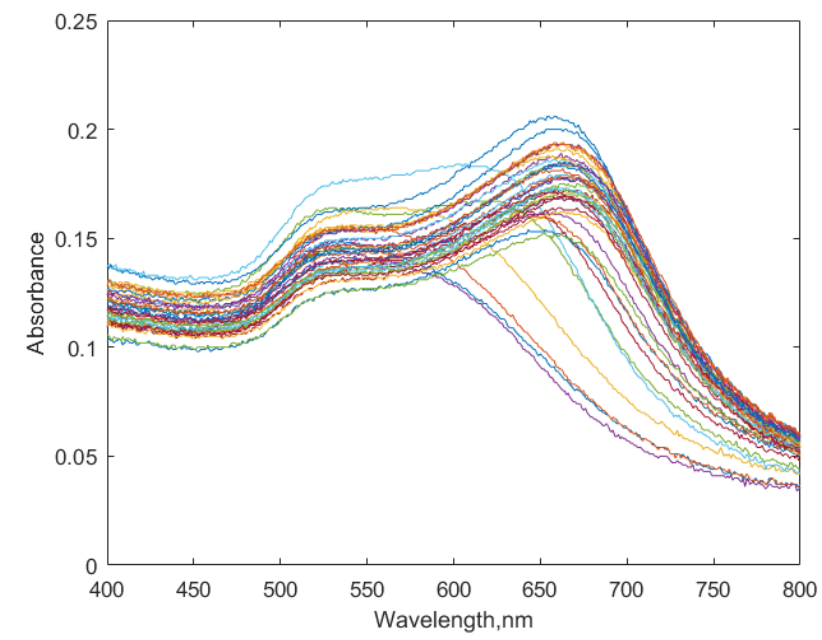

Fig. 7. UV-visible spectra of gold nanoparticles with Bismuthiol II solution after the addition different concentrations of mercury ions without pretreatment 


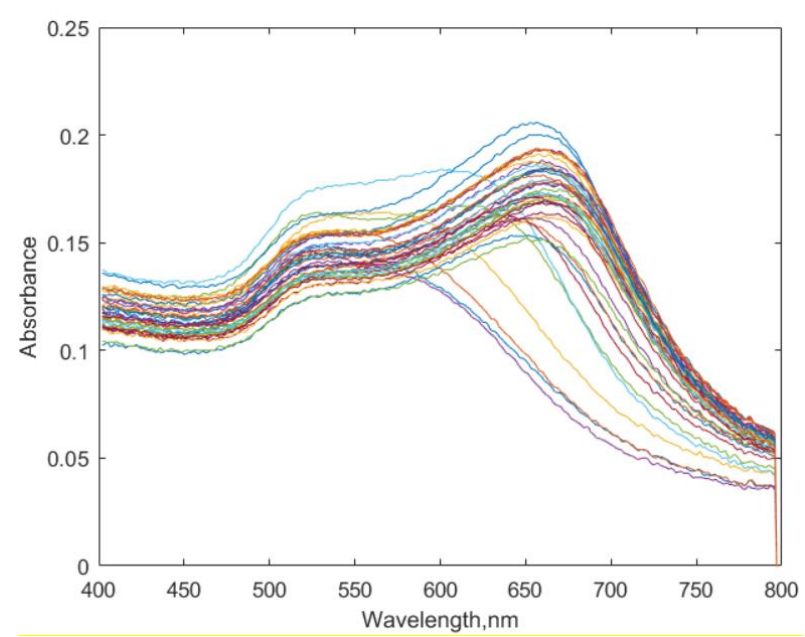

Fig. 8. The results of Savitzky-Golay convolution smoothing

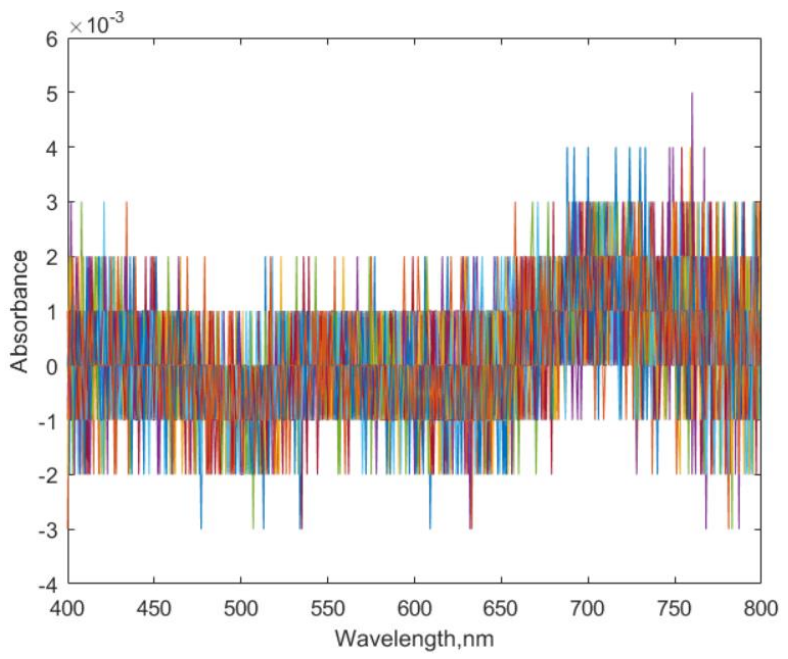

Fig. 9. The results of first-order derivative

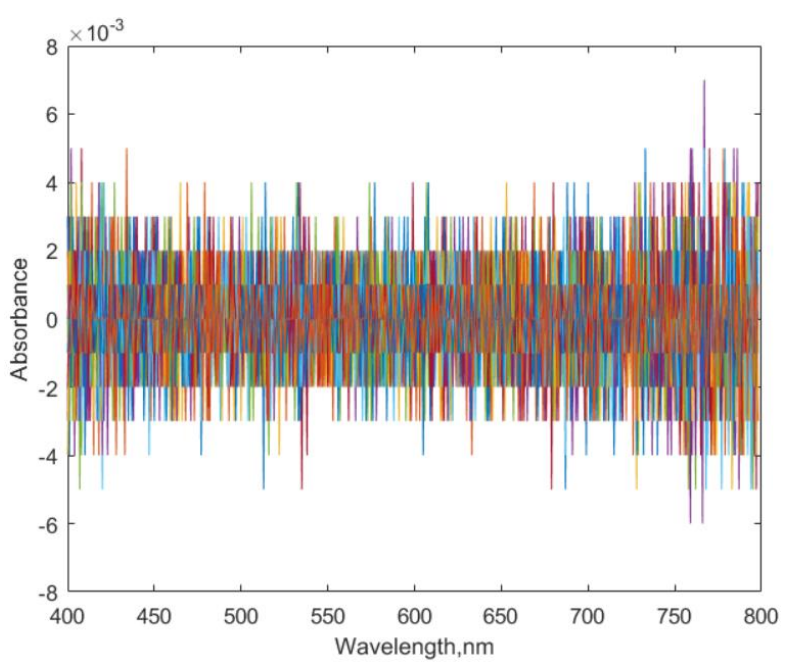

Fig. 10. The results of second-order derivative

Table 1 shows the results of different pretreatment methods for partial least squares models. The table employs 4 indexes to evaluate model accuracy, including the correlation coefficients for the calibration set $(R \mathrm{c})$, the correlation coefficients for prediction set $(R \mathrm{p})$, the root mean square error of calibration for the calibration set (RMSEC), and the root mean square error of prediction for the prediction set (RMSEP).

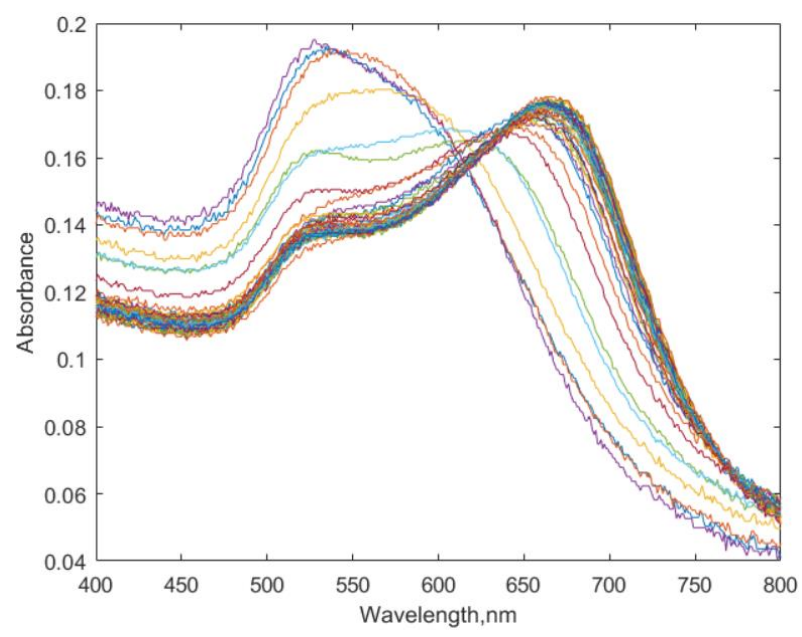

Fig. 11. The results of multiplicative scatter correction

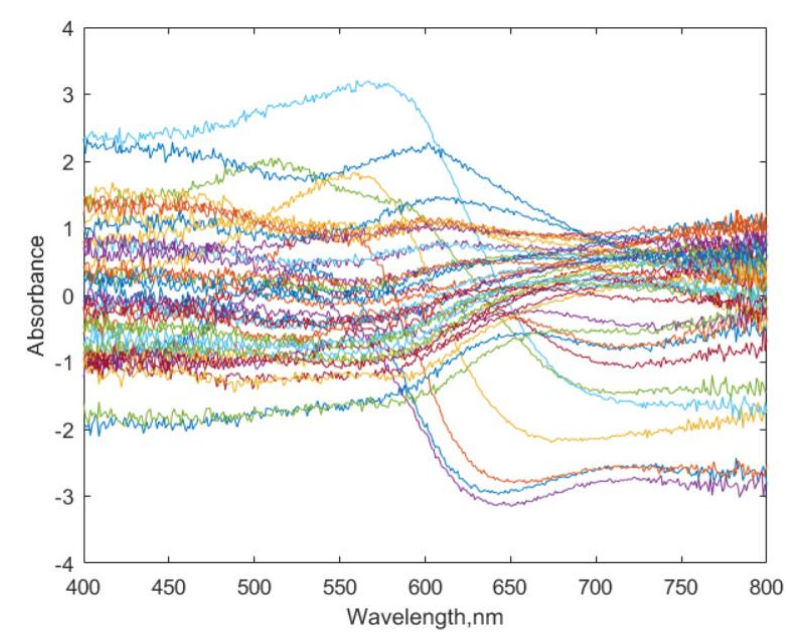

Fig. 12. The results of standard normal variete

Table 1. Comparisom of different data pretreatment methods for partial least squares models. Correlation coefficients for calibration set, correlation coefficients for prediction set, root mean square error of prediction set are used to compare the performance of models when using different pretreatment methods

\begin{tabular}{|c|c|c|c|c|c|}
\hline \multirow{2}{*}{$\begin{array}{l}\text { Pretreatment } \\
\text { method }\end{array}$} & \multirow{2}{*}{$\begin{array}{c}\text { PLS } \\
\text { principal } \\
\text { component }\end{array}$} & \multicolumn{2}{|c|}{ Calibration set } & \multicolumn{2}{|c|}{ Prediction set } \\
\hline & & $R \mathrm{c}$ & RMSEC & $R \mathrm{p}$ & RMSEP \\
\hline $\begin{array}{l}\text { No } \\
\text { pretreatment } \\
\text { (original data) }\end{array}$ & 2 & 0.9274 & 56 & 0.9226 & 0.1665 \\
\hline $\begin{array}{l}\text { SG } \\
\text { convolution } \\
\text { smoothing }\end{array}$ & 2 & 0.9270 & 0.1160 & 0.9227 & 0.1662 \\
\hline $\begin{array}{l}\text { First-order } \\
\text { Derivative }\end{array}$ & 6 & 0.9835 & 0.0559 & 0.7589 & 0.1762 \\
\hline $\begin{array}{l}\text { Second-order } \\
\text { Derivative }\end{array}$ & 1 & 0.4893 & 0.2697 & 0.4365 & 0.242 \\
\hline MSC & 1 & 0.9352 & 0.1095 & 0.9381 & 0.1637 \\
\hline SNV & 3 & 0.9319 & 0.1121 & 0.9076 & 0.1148 \\
\hline
\end{tabular}

SG: Savitzky-Golay

MSC: Multiplicative scatter correction

SNV: Standard Normal Variete

PLS: partial least squares

PLS: Partial Least Squares

Rc: correlation coefficients for calibration set

Rp: correlation coefficients for prediction set

RMSEC: Root Mean Square Error of Calibration set

RMSEP: Root Mean Square Error of Prediction set 
The correlation coefficient represents the related degree between the two variables in the prediction set and calibration set; the closer and smaller the RMSEC and RMSEP are, the better the forecast prediction model [21].

Table 1 compares the forecast model indexes using different pretreatment methods. The RMSEP for the prediction set after the standard normal variate method processing is the smallest, which is close to RMSEC for the calibration set, $0.1121 \mathrm{mg} / \mathrm{L}$ and $0.1148 \mathrm{mg} / \mathrm{L}$, respectively; at the same time; the $R \mathrm{c}$ and $R \mathrm{p}$ exceed 0.9 , demonstrating that the models using the standard normal variate method pretreatment had the best prediction effect. Therefore, in this study, the standard normal variate method was adopted for preprocessing the spectral data before the models were built.

\subsection{Prediction models of mercury ions}

\subsubsection{Principal component analyses (PCA)}

Principal Component Analyses can reduce dimensionality by establishing as few as possible new variables that are irrelevant in pairs and remain the original useful information based on the original variables. Firstly, several principal components must be chosen by calculating and ranking the variance contribution rate. Table 2 shows the sum of the variance contribution rate of the first 5 principal components with increasing order. The sum of the variance contribution rate of the first 3 principal components reached more than $95 \%$. Consequently, the first 3 principal components were chosen to build a linear regression model.

Table 2. Principal components variance contribution rate. Variance contribution rate is calculated with different principal component number

\begin{tabular}{|c|c|}
\hline Principal component number & Variance contribution rate, $\%$ \\
\hline 1 & 53.25904 \\
\hline 2 & 88.74301 \\
\hline 3 & 95.45907 \\
\hline 4 & 96.59044 \\
\hline 5 & 97.70781 \\
\hline
\end{tabular}

Table 3 illustrates the results of the principal component analyses regression. The $R \mathrm{p}$ of the samples for the prediction set was greater than 0.9 , while $R \mathrm{c}$ was 0.9235. Additionally, RMSEC and RMSEP were small and close to each other at $0.1187 \mathrm{mg} / \mathrm{L}$ and $0.1139 \mathrm{mg} / \mathrm{L}$ respectively, demonstrating that the model is highly accurate.

Table 3. Results of principal component analyses regression. Correlation coefficients for calibration set, correlation coefficients for prediction set, correlation coefficients for prediction set and root mean square error of prediction set are used to evaluate the accuracy of principal component analyses regression

\begin{tabular}{|l|c|c|c|c|c|c|}
\hline $\begin{array}{l}\text { Detection } \\
\text { object }\end{array}$ & \multicolumn{3}{|c|}{ Calibration set } & \multicolumn{3}{c|}{ Prediction set } \\
\hline \multirow{2}{*}{ Mercury ions } & $R \mathrm{c}$ & $R \mathrm{c}^{2}$ & RMSEC & $R \mathrm{p}$ & $R \mathrm{p}^{2}$ & RMSEP \\
\cline { 2 - 7 } & 0.9235 & 0.8528 & 0.1187 & 0.9085 & 0.8254 & 0.1139 \\
\hline
\end{tabular}

\subsubsection{Full bands partial least squares regression analysis}

A full band partial least squares model was built after the standard normal variate pretreatment; and three principal components were determined via the cross validation method. $R \mathrm{c}^{2}$ was 0.9077 with $R \mathrm{p}^{2} 0.8238$, as shown in table 4, while the RMSEC and RMSEP were $0.1122 \mathrm{mg} / \mathrm{L}$ and $0.1148 \mathrm{mg} / \mathrm{L}$, respectively, revealing the good predictive ability of this model.

Table 4. Results of full band partial least squares model

\begin{tabular}{|l|c|c|c|c|c|c|}
\hline $\begin{array}{l}\text { Detection } \\
\text { object }\end{array}$ & \multicolumn{3}{|c|}{ Calibration set } & \multicolumn{3}{|c|}{ Prediction set } \\
\hline \multirow{2}{*}{ Mercury ions } & $\mathrm{Rc}$ & $\mathrm{Rc}^{2}$ & $\mathrm{RMSEC}$ & $\mathrm{Rp}$ & $\mathrm{Rp}^{2}$ & $\mathrm{RMSEP}$ \\
\cline { 2 - 7 } & 0.9319 & 0.8684 & 0.1122 & 0.9077 & 0.8238 & 0.1148 \\
\hline
\end{tabular}

\subsubsection{Feature bands partial least squares regression analysis}

Since full bands have a large amount of data that must be processed, a quantitative calibration model built by selecting a feature band is preferable, as it could reduce calculation time and remove unwanted variables to obtain better predictive ability.

Fig. 13 shows the negative correlation coefficient between the concentration of mercury ions and absorbance. Spectral data from $620 \mathrm{~nm}$ to $800 \mathrm{~nm}$ was chosen to the build partial least squares model, which was preprocessed using a standard normal variate method as well. The number of principal factors of the model was determined to be 4 by cross-validation method.

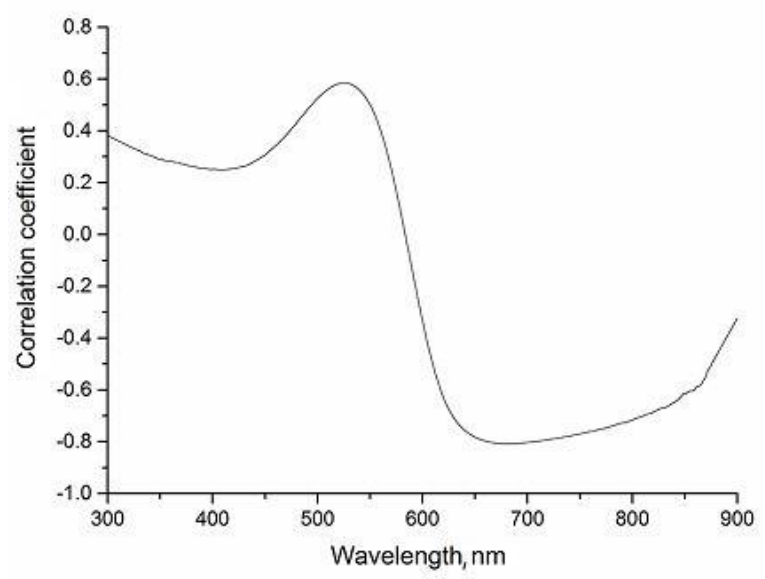

Fig. 13. The correlation coefficient between the concentration of mercury ions and absorbance

Table 5 shows the modeling results. Compared with the full bands partial least squares model, $R \mathrm{c}$ and $R \mathrm{p}$ were slightly larger, suggesting the higher relevance of the model; RMSEC and RMSEP were smaller and closer at $0.0986 \mathrm{mg} / \mathrm{L}$ and $0.1020 \mathrm{mg} / \mathrm{L}$, respectively, which demonstrates higher precision and better predictive ability.

Table 5. Results of relevant band partial least squares model

\begin{tabular}{|l|c|c|c|c|c|c|}
\hline $\begin{array}{l}\text { Detection } \\
\text { object }\end{array}$ & \multicolumn{3}{|c|}{ Calibration set } & \multicolumn{3}{|c|}{ Prediction set } \\
\hline \multirow{2}{*}{ Mercury ions } & $R \mathrm{c}$ & $R \mathrm{c}^{2}$ & RMSEC & $R \mathrm{p}$ & $R \mathrm{p}^{2}$ & RMSEP \\
\cline { 2 - 7 } & 0.9477 & 0.8983 & 0.0986 & 0.9274 & 0.8601 & 0.1020 \\
\hline
\end{tabular}




\subsection{Comparison of results}

In this study, principal component regression analysis and partial least squares regression analysis were employed to build mercury ion concentration forecast models. By analyzing the results of different models, a linear relationship between the concentration and spectrum was obtained, indicating that it is feasible for the colorimetric method using gold nanoparticles to detect $\mathrm{Hg}^{2+}$. Compared to other models, the partial least squares model is more reliable, especially in the partial least squares model in the feature bands, where the $\mathrm{Rc}$ and $\mathrm{Rp}$ are highest, the standard deviation is lowest, and the predictive ability is optimal.

\subsection{Selectivity of gold nanoparticles and practical application}

To study the selectivity of this colorimetric sensor, several competitive metal ions involving Manganese ions $\left(\mathrm{Mn}^{2+}\right)$, Copper ions $\left(\mathrm{Cu}^{2+}\right)$, Magnesium ions $\left(\mathrm{Mg}^{2+}\right)$, Zinc ions $\left(\mathrm{Zn}^{2+}\right)$, Sodium ions $\left(\mathrm{Na}^{+}\right)$, Calcium ions $\left(\mathrm{Ca}^{2+}\right)$, Iron ions $\left(\mathrm{Fe}^{3+}\right)$, Lead ions $\left(\mathrm{Pb}^{2+}\right)$, Silver ions $\left(\mathrm{Ag}^{+}\right)$, Nickel ions $\left(\mathrm{Ni}^{2+}\right)$ and Cadmium ions $\left(\mathrm{Cd}^{2+}\right)$ at a concentration of $0.3 \mathrm{mg} / \mathrm{L}$, which was 20 times higher than mercury ions, and water in the river were added to the GNPs-Bismuthiol II solution. The result indicated that the intensity of the surface plasmon resonance bands of other metals showed a slight change compared to the blank one, as shown in Fig. 14. And the solution color remained constant when the other metal ions were added, while the color changed to red after the addition of $\mathrm{Hg}^{2+}$. Fig. 14 also shows that the $\mathrm{Hg}^{2+}$ intensity gap is most obvious at $650 \mathrm{~nm}$ and $530 \mathrm{~nm}$ absorbance for various metals and river water. Table 6 shows the absorbance ratio (A650/A530) of mercury ions, other metal ions and river water; other metal ions and river water showed significant values and more than the value of $\mathrm{Hg}^{2+}$ which is 0.0672 . Hence, this colorimetric method can be used to detect mercury ions with high selectivity. This method was also tested in tap water, and the lowest detectable concentration of $\mathrm{Hg}^{2+}$ was estimated to be $0.01146 \mathrm{mg} / \mathrm{L}$.

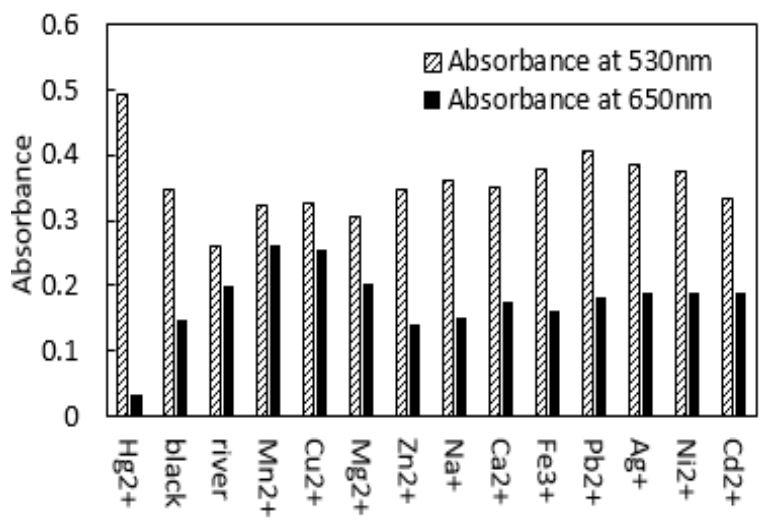

Fig. 14. The absorbance at $530 \mathrm{~nm}$ and $650 \mathrm{~nm}$ of gold nanoparticles with Bismuthiol II solution upon the addition of mercury ions and other metal ions

In order to confirm the reliability of the colorimetric method, the same concentration of mercury ions were added to the GNPs-Bismuthiol II solution, respectively.
The partial least square model was used to analysize the collected spectral data, whose results are shown in Table 7. The average recovery was $101.8 \%$, that was in the range of $95 \%-105 \%$, indicating that the method was reliable.

Table 6. Comparison of absorbance ratio value (A650/A530) at $650 \mathrm{~nm}$ and $530 \mathrm{~nm}$ of gold nanoparticles with Bismuthiol II solution upon the addition of mercury ions and other metal ions

\begin{tabular}{|c|c|}
\hline Heavy metal ions & Ratio value of A650/A530 \\
\hline Mercury Ions & 0.0672 \\
\hline Blank & 0.4259 \\
\hline River water & 0.7698 \\
\hline Manganese ions & 0.8184 \\
\hline Copper ions & 0.7821 \\
\hline Magnesium ions & 0.6630 \\
\hline Zinc ions & 0.3984 \\
\hline Sodium ions & 0.4162 \\
\hline Calcium ions & 0.5008 \\
\hline Iron ions & 0.4300 \\
\hline Lead ions & 0.4521 \\
\hline Silver ions & 0.4926 \\
\hline Nickel ions & 0.5059 \\
\hline Cadmium ions & 0.5671 \\
\hline
\end{tabular}

Table 7. Results of mercury ion parallel experimental

\begin{tabular}{|c|c|c|c|}
\hline $\begin{array}{c}\text { The number of } \\
\text { measurements }\end{array}$ & $\begin{array}{c}\text { Spiked } \\
\text { concentration, } \\
\mathrm{mg} / \mathrm{L}\end{array}$ & $\begin{array}{c}\text { The average } \\
\text { measured value, } \\
\mathrm{mg} / \mathrm{L}\end{array}$ & $\begin{array}{c}\text { Average } \\
\text { recovery, } \%\end{array}$ \\
\hline 5 & 0.3000 & 0.3114 & 103.8 \\
\hline 5 & 0.6000 & 0.5988 & 99.8 \\
\hline
\end{tabular}

\section{CONCLUSIONS}

In summary, this study developed a simple, quick, easy-to-use, highly selective colorimetric method for detecting $\mathrm{Hg}^{2+}$ in aqueous media using gold nanoparticles with a suitable forecast model. Adding $\mathrm{Hg}^{2+}$ to the gold nanoparticles coated with Bismuthiol II changed the gold nanoparticles color and absorbance with the aggregation state of gold nanoparticles. The principal component analyses, full bands partial least squares regression, and relevant bands partial least squares regression were employed to build models to find the best forecast model based on the UV-Vis spectral data processing after the colorimetric reaction. In the best forecast model, that is, the relevant bands partial least squares model, $R \mathrm{c}$ and $R \mathrm{p}$ were 0.9477 and 0.9274 , respectively, while the RMSEC and RMSEP were $0.0986 \mathrm{mg} / \mathrm{L}$ and $0.1020 \mathrm{mg} / \mathrm{L}$, respectively. In addition, this colorimetric method exhibited excellent selectivity through adding several competitive metal ions to the GNPs-Bismuthiol II solution. These results show that spectroscopy combined with the colorimetric method using gold nanoparticles was successfully applied for detecting mercury ions in an aqueous media. Hence, it has a great potential application in the area of water quality monitoring and environmental pollution analysis.

\section{Acknowledgments}

Funding for this research is provided by the International Science and Technology Cooperation 
Program of China (No.2013DFA11320), Shandong province specific project for independent innovation and achievements transformation (No.2014ZZCX07102), and Development of the decision-making system in agricultural non-point source and heavy metal pollution management (No.2016YFD0800907).

\section{REFERENCES}

1. Selid, P.D., Xu, H., Collins, E.M., Facecollins, M.S., Zhao, J.X. Sensing Mercury for Biomedical and Environmental Monitoring Sensors 9(7) 2009: pp. 5446-5459. https://doi.org/10.3390/s90705446

2. Gao, S., Jia, X., Chen, Y. Old Tree with New Shoots: Silver Nanoparticles for Label-free and Colorimetric Mercury Ions Detection Journal of Nanoparticle Research 15 (1) 2013: pp. $1-9$.

https://doi.org/10.1007/s11051-012-1385-4

3. World Health Organization. Guidelines for Drinking-water Quality. 2011. Geneva: WHO.

4. Burda, C., Chen, X., Narayanan, R., El-Sayed, M.A. Chemistry and Properties of Nanocrystals of Different Shapes Chemical Reviews 105 (4) 2005: pp. 1025-1102. https://doi.org/10.1002/chin.200527215

5. Shukla, R., Bansal, V., Chaudhary, M., Basu, A., Bhonde, R.R., Sastry, M. Biocompatibility of Gold Nanoparticles and Their Endocytotic Fate Inside the Cellular Compartment: A Microscopic Overview Langmuir the ACS Journal of Surfaces \& Colloids 21 (23) 2005: pp. $10644-10654$. https://doi.org/10.1021/la0513712

6. Daniel, M. C., Astruc, D. Gold Nanoparticles: Assembly, Supramolecular Chemistry, Quantum-Size-Related Properties, and Applications Toward Biology, Catalysis, and Nanotechnology Chemical Reviews 104 (1) 2004: pp. $293-346$. https://doi.org/10.1021/cr030698+

7. Ghosh, S.K., Pal, T. Interparticle Coupling Effect on the Surface Plasmon Resonance of Gold Nanoparticles: From Theory to Applications Chemical Reviews 107 (11) 2007: pp. $4797-4862$. https://doi.org/10.1021/cr0680282

8. Knecht, M.R., Sethi, M. Bio-Inspired Colorimetric Detection of $\mathrm{Hg} 2+$ and $\mathrm{Pb} 2+$ Heavy Metal Ions Using $\mathrm{Au}$ Nanoparticles Analytical \& Bioanalytical Chemistry 394 (1) 2009: pp. 33-46. https://doi.org/10.1007/s00216-008-2594-7

9. Starace, A.F. Atomic spectroscopy (Book Reviews: The Theory of Atomic Structure and Spectra) Science 216(4542) 1982: pp. 169-170. https://doi.org/10.1126/science.216.4542.169

10. Butler, O.T., Cairns, W.R.L., Cook, J.M., Davidson, C.M. Atomic Spectrometry Update. Environmental Analysis Journal of Analytical Atomic Spectrometry $25(2)$ 2010: pp. $103-141$.

https://doi.org/10.1039/b925232k
11. Evans, E.H., Day, J.A., Palmer, C.D., Smith, C.M.M. Atomic Spectrometry Update. Advances in Atomic Spectrometry and Related Techniques Journal of Analytical Atomic Spectrometry 24 (6) 2009: pp. 711-733. https://doi.org/10.1039/b907325f

12. Chai, F., Wang, C., Wang, T., Li, L., Su, Z. Colorimetric Detection of $\mathrm{Pb} 2+$ Using Glutathione Functionalized Gold Nanoparticles ACS Applied Materials \& Interfaces 2 (5) 2010: pp. $1466-1470$. https://doi.org/10.1021/am100107k

13. Lee, H.M., Cho, S.W., Kim, J.H., Kim, C.K. Electrochemical Analysis of the Electrodeposition of Platinum Nanoparticles Korean Chemical Engineering Research 53 (5) 2015: pp. 540-544. https://doi.org/10.9713/kcer.2015.53.5.540

14. Suprun, E.V., Zaryanov, N.V., $\quad$ Radko, S.P., Kulikova, A.A., $\quad$ Kozin, S.A., $\quad$ Makarov, A.A., Archakov, A.I., Shumyantseva, V.V. Tyrosine Based Electrochemical Analysis of Amyloid- $\beta$ Fragment $(1-16)$ Binding to Metal (II) Ions Electrochimica Acta 179 2015: pp. $93-99$. https://doi.org/10.1016/j.electacta.2015.01.066

15. Xu, S.H., Zhu, Y.P., Xiong, D.Y., Wang, L.W., Yang, P.X., Chu, P.K. Electrochemical Analysis of Interface Adsorption Phenomena on Three-dimensional Nano-nickel Electrode Deposited on Silicon Microchannel Plate Electrochimica Acta 194 2016: pp. 253-262. https://doi.org/10.1016/j.electacta.2016.02.131

16. Stevaert, A., Nurra, S., Pala, N., Carcelli, M., Rogolino, D., Shepard, C., Domaoal, R.A., Kim, B., Alfonso-Prieto, M., Marras, S.A.E., Sechi, M., Naesens, L. An Integrated Biological Approach to Guide the Development of Metal-chelating Inhibitors of Influenza Virus PA Endonuclease Molecular Pharmacology 87 (2) 2014: pp. $323-337$. https://doi.org/10.1124/mol.114.095588

17. Chihomvu, P., Stegmann, P., Pillay, M. Characterization and Structure Prediction of Partial Length Protein Sequences of PcoA, PcoR and ChrB Genes from Heavy Metal Resistant Bacteria from the Klip River, South Africa International Journal of Molecular Sciences $16(12)$ 2015: pp. $7352-7374$. https://doi.org/10.3390/ijms 16047352

18. Chu, X.L. Chemometrics and Molecular Spectroscopy Technology. Chemical Industry Press: Beijing, China, 2011.

19. Lu, W.Z. Modern Near Infrared Spectroscopy. China Petrochemical Press: Beijing, China, 2007.

20. Duan, J., Yang, M., Lai, Y.C., Yuan, J.P., Zhan, J.H. A Colorimetric and Surface-Enhanced Raman Scattering DualSignal Sensor for $\mathrm{Hg} 2+$ Based on Bismuthiol II-Capped Gold Nanoparticles Analytica Chimica Acta 723 2012: pp. $88-93$. https://doi.org/10.1016/j.aca.2012.02.031

21. Li, M.Z. Spectroscopy Technique and Application. Science Press: Beijing, China, 2006. 